\title{
LA FILOSOFÍA LEIBNIZIANA DE LA SUBJETIVIDAD
}

\section{THE LEIBNIZIAN PHILOSOPHY OF SUBJECTIVITY}

\author{
AdELINO CARDOSO ${ }^{1}$ \\ Facultade de Ciências Sociais e Humanas \\ Universidade Nova de Lisboa
}

Resumen: Leibniz elabora una filosofía original de la subjetividad, formada en la confrontación con el cogito cartesiano y con los autores que asumen el legado de Descartes, en especial con Arnauld. Para Leibniz, "yo pienso" es una proposición cierta y evidente, pero no una verdad universal de razón, que pueda servir de fundamento a todo saber. Se trata de una verdad de hecho, a la cual corresponde una experiencia insuperablemente confusa, que apela a un modo especial de inteligibilidad. En este marco, Leibniz delinea una gramática del yo, entendido como una estructura relacional, una expresión singular del mundo común.

En la fase monadológica, que culmina el ejercicio filosófico leibniziano, la subjetividad se identifica con el dinamismo espontáneo de la vida perceptiva, desarrollada bajo la forma de un dinamismo espontáneo subconsciente, a nivel de lo primordial. Por lo que, sin llegar a ser su estrato originario, la conciencia representa una expresión más elevada del dinamismo perceptivo, garantizando la identidad del yo percipiente.

\footnotetext{
${ }^{1}$ CHAM, Universidade Nova de Lisboa, Facultade de Ciências Sociais e Humanas, Av. Berna, 26-C, 1069-061 Lisboa, Portugal. E-amil: cardoso.adelino@gmail.com. Traducción del original al castellano por Letícia Cabañas.
} 
Palabras Clave: cogitatio, mundo, percepción, vida, yo.

AвSTRACT: Leibniz works out an original philosophy of subjectivity, which is formed in confrontation with the Cartesian cogito, alongside authors who take up Descartes's legacy, namely the influential Arnauld. For Leibniz, the proposition "I think" is certain and evident; however, it is not a universal truth of reason, that could be the very foundation of all knowledge. It is a truth of fact, to which an insurmountably confused experience corresponds, which appeals to a special way of intelligibility. Within this framework, Leibniz delineates a grammar of the I, viewed as a relational structure, a singular expression of our shared world.

In the Leibnizian philosophical exercise, which culminates in the monadological system, subjectivity is interpreted as subconscious spontaneous dynamism, at the level of a primordial Self. Therefore, consciousness is the highest expression of the perceptive dynamism, through which the identity of the percipient subject is assured, although it is not the primary stratum of perception.

KEYwORDs: cogitatio, I, life, perception, world.

\section{La reinterpretación leibniziana de la subjetividad}

El objetivo principal de este trabajo consiste en caracterizar la filosofía leibniziana de la subjetividad y determinar su lugar en el marco de la modernidad, entendida ésta como un espacio plural en donde se confrontan múltiples planteamientos y doctrinas.

Sin duda el cogito cartesiano ocupa un lugar matricial en la elaboración de la subjetividad moderna, convirtiéndose en referencia ineludible para los pensadores de la segunda mitad del siglo XVII. Llegó a suscitar un vivo debate, incluso entre autores seguidores del legado cartesiano. Un ejemplo es la controversia entre Arnauld y Malebranche sobre las ideas. Por su parte, E. Husserl, el fundador de la fenomenología, reconoce en el cogito una fuente de inspiración y reflexión, localizándose allí el inicio de la fenomenología.

En el marco del pensamiento cartesiano, el cogito responde a la exigencia de "un punto de apoyo firme e inmóvil" (punctum quod esset firmum et immobile) 
que sirva de fundamento "incuestionable" para todo saber, gracias a su estatuto de verdad caracterizada como "la más cierta y más evidente de todas" (omnium certissimam evidentissimamque $)^{2}$.

El significado principal de la cogitatio es de índole intelectual, por consistir predominantemente en concebir y juzgar, pero abarca también el ámbito de la sensibilidad: "es cierto que me parece que veo luz, que oigo ruido y que siento calor; esto no puede ser falso y esto es, propiamente, lo que en mí se llama sentir y esto, precisamente, es pensar" ${ }^{3}$. La experiencia de lo que sucede en sí mismo "no puede ser falsa", al ir acompañada por un modo especial de evidencia, tal como se afirma en el artículo 26 de Las pasiones del alma:

Debe destacarse también que a veces sucede que esa pintura es tan semejante a la cosa representada, que uno puede engañarse respecto de las percepciones referidas a lo objetos que existen fuera de nosotros, o bien las que se refieren a algunas partes de nuestro cuerpo ; pero que no puede engañarse de la misma manera en lo tocante a las pasiones, pues le son tan cercanas e íntimas a nuestra alma que es imposible que las sienta sin que sean verdaderamente tal y como las siente. ${ }^{4}$

El sentimiento interior es intrínsecamente verdadero debido a su inmediatez y modalidad ontológica propias: la alegría no es otra cosa que estar alegre, sentir alegría.

Algunos pensadores inspirados directamente en Descartes asumen el cogito como el sentimiento de sí mismo. Es el caso, entre otros, de La Forge ${ }^{5}$ y Malebranche. Este último se ve forzado a romper con la cadena cartesiana de razones, al considerar que, lejos de ser la cosa más fácil de conocer, el alma no es susceptible de un auténtico conocimiento: "La ilusión de que se conoce el alma es uno

\footnotetext{
2 Descartes, Meditationes de prima philosophia, AT VII, 24-25 ; trad. M. García Morente, Madrid, Encuentro, 2005, pp. 154-155.

${ }^{3}$ Ibid., p. 29 ; trad. ibid. p. 159.

${ }^{4}$ Descartes, AT XI, 348 ; trad. J.A. Martínez y P. Andrade, Madrid, Tecnos, 2a ed., 2006, pp. 94-95.

${ }^{5} \mathrm{Al}$ referirse a la naturaleza del espíritu como sustancia pensante, este cartesiano define el pensamiento: "entiendo aquí por Pensamiento esa percepción, conciencia o conocimiento interior que cada uno de nosotros siente inmediatamente por sí mismo, al apercibirse de lo que hace o de lo que en él sucede" (La Forge, 1664, 14).
} 
de nuestros más vivos sentimientos, dando lugar a muchos prejuicios"' ${ }^{\text {. Para un }}$ filósofo oratoriano, conciencia y evidencia no concuerdan bien entre sí.

En la fase inicial de su elaboración filosófica, Leibniz asume el cogito como un sentimiento interno, cuya verdad se funda en su inmediatez. Se expresa en términos muy próximos a Descartes: "Aquello que sentimos que pensamos o que no pensamos lo sentimos verdaderamente. Pues el sentir (sensio) del pensamiento es inmediato al propio pensamiento en el mismo sujeto, y por tanto no hay ninguna causa de error"7. De ahí la tesis general de que "todo sentimiento de nuestro pensamiento es verdadero"s.

La carta a Jean Gallois, de fines de 1672, es sumamente interesante en lo que se refiere al significado del cogito. En su intento de ofrecer una respuesta a las objeciones planteadas por el escepticismo, Leibniz afirma la existencia de proposiciones evidentes, esto es, que no admiten demostración?. Tales proposiciones son de dos tipos, sensibles e intelectuales:

En primer lugar deben admitirse aquellas [verdades] de las que tenemos constancia por los sentidos, como, por ejemplo, que yo me siento a mí mismo como sentiente; pero también aquellas que, conocidas mediante los sentidos, se demuestran empleando definiciones, como son las que se demuestran en base al anterior: 'yo me siento a mí mismo' o pienso [...] Igualmente habrá que recoger las proposiciones idénticas o bien la afirmación de lo mismo por sí mismo, con las mismas palabras" (A II 1, 227 [A II, $\left.\left.1^{2}, 350\right]\right)$.

El orden del texto resulta significativo. Se trata claramente de un orden psicológico, quoad nos: en primer lugar las verdades sensibles y a continuación las inteligibles. A su vez, las verdades sensibles se dividen en dos niveles: aquellas

\footnotetext{
${ }^{6}$ Malebranche, 1974 , pp. 30-31. La viveza del sentimiento es señal de su opacidad: "Cuando más vivos son los sentimientos, más tinieblas siembran" (Conversaciones sobre la metafísica y la religión, trad..P. Andrade e I. Quintanilla, Madrid, Encuentro, 2006, p. 65).

7 Specimen demonstrationum, 1671, A VI 2, 305.

${ }^{8}$ Ibid, A VI 2, 307.

${ }^{9}$ Fernando Gil, que clasifica a la evidencia como "verdad redoblada", muestra acertadamente que hay un régimen de lenguaje propio de la evidencia, que encaja en el sistema percepciónlenguaje. (GIL, 1996, 53-83).
} 
que son en cierto modo principales, esto es, que no dependen de ninguna otra y son fuente de nuevas verdades, y las que de ellas dependen. A las primeras corresponde lo que podemos designar como puro fenomenalismo, la experiencia inmediata de sí mismo en cuanto sentiente. Nos situamos en el plano de la facticidad, del acontecer. Las segundas, tales como "siento", "pienso", "soy", no son inmediatamente sensibles, sino inferencias que resultan de las primeras. Por consiguiente, la evidencia racional sensible no se refiere al cogito como certeza de la existencia del ser pensante en cuanto tal, sino al pensamiento dado en acto. Leibniz lo explica de un modo muy preciso:

Resulta cierto por los sentidos que yo me siento como sentiente; por lo tanto, que el 'yo sentiente' se siente inmediatamente o sin mediación, ya que entre yo y yo en la mente no hay nada intermedio. Lo que se siente inmediatamente es inmediatamente sensible. Lo que es inmediatamente sensible es sensible sin posibilidad de error (porque todo error proviene de la mediación sensible, tal como supongo que debe ser demostrado en otro lugar) (Ibid.).

El léxico de Leibniz sufre transformaciones, pero permanece constante la visión de un cogito sensible, carente de toda pretensión de fundamento y universalidad. Así, en los Nuevos Ensayos, donde el yo pienso es asumido como una verdad primera de hecho:

Siempre se puede afirmar que la proposición "yo existo" es plenamente evidente, por ser una proposición que no puede ser demostrada a partir de otra, o bien una verdad inmediata. Y decir "pienso, luego existo" no supone propiamente demostrar la existencia partiendo del pensamiento, puesto que pensar y ser pensante son la misma cosa, y decir "soy pensante" ya es decir "soy". Sin embargo, esta proposición la podéis excluir de los axiomas con cierta razón, ya que se trata de una proposición de hecho, basada en una experiencia inmediata, y no es una proposición necesaria. ${ }^{10}$

${ }^{10}$ NE IV, vii, 7, GP V, 391-392; trad. J. Echeverría, Madrid, Alianza, 1992, p. 490. 
Leibniz reformula su tesis en el sentido de que las locuciones 'pienso, existo' se infieren de la experiencia inmediata de sí, por considerar que el existir y el pensar son parte intrínseca de esa experiencia, que es máximamente evidente, aunque contingente. Se plantea la cuestión acerca del contenido de esa evidencia. Ahora bien, la evidencia se refiere únicamente al hecho de que yo existo y no a aquello que sobrepasa a ese mismo hecho, es decir, lo que yo soy, mi naturaleza. Atendiendo a la gnoseología leibniziana, y en especial a las Meditaciones, surge la pregunta: ¿la experiencia de nosotros mismos como seres pensantes es un conocimiento distinto? La respuesta resulta ser que tal experiencia es inevitablemente confusa: "No es suficiente que yo me sienta como una sustancia que piensa; sería necesario concebir claramente lo que me distingue de todos los demás espiritus, pero sólo tengo de ello una experiencia confusa." ${ }^{11}$

Naturalmente Leibniz no ignora la posición de Arnauld a este respecto, expresada en la controversia con Malebranche: "Hay razones para creer que Dios, al crear el alma le dio la idea de sí misma, y que posiblemente ese pensamiento de sí misma constituye su esencia." ${ }^{12} \mathrm{El}$ interés de Leibniz por enfrentarse a la tesis de la autotransparencia de la conciencia está plenamente justificado debido a la relevancia de la cuestión en debate.

Ciertamente no es algo acccidental que en esta ocasión el interlocutor de Leibniz sea el filósofo que declara expresamente que el cogito implica la visión inmediata de la propia esencia.

\section{Reformulación de la noción del individuo como un yo}

Arnauld se propone debatir con Leibniz la noción de sustancia formulada en el Discurso de Metafisica, considerando que la clave se encuentra en la noción del yo. Así, oponiéndose a la doctrina malebranchiana de la visión en Dios, afirma el insigne teólogo: "todo lo que quiero concluir, digo, es que no es en Dios, que habita en una luz inaccesible para nosotros, donde debemos ir a buscar las verdaderas nociones, específicas o individuales, de las cosas que conocemos, sino

${ }^{11}$ Observaciones de Leibniz a una carta de Antoine Arnauld, 14. 07. 1686, OFC 14, pp. 42-43. ${ }^{12}$ Arnauld, 1986, p. 260. 
en las ideas que encontramos en nosotros mismos. Ahora bien, yo encuentro en mí la noción de una naturaleza individual, ya que encuentro la noción de 'yo'."13

Desde el punto de vista metodológico, la ciencia del yo o del espíritu opera de igual modo que la ciencia geométrica de la naturaleza: partiendo de ideas simples y bien definidas, como ya quedó claramente indicado en La logique, ou l'art de penser. En efecto, el "único principio" de todo saber es éste: "Que todo lo que está contenido en la idea verdadera de una cosa (es decir, en la percepción clara que tenemos de ella), puede ser afirmado como verdadero" ${ }^{14}$. Por consiguiente, la ciencia del espíritu debe seguir el modelo axiomático-deductivo de la matemática. Así, el autor asume el modus operandi de la geometría para conocer las propiedades de la esfera y concluye : "Aplico la misma regla a la noción individual de 'yo'” (OFC 14, 30). Aquí se localiza el núcleo de la divergencia entre Leibniz y Arnauld.

Según Leibniz, existe una gramática propia del yo y, por consiguiente, "es necesario filosofar de otra manera acerca de la noción de una sustancia individual que de la noción específica de la esfera." ${ }^{15}$ ¿En qué consiste tal diferencia y cuál es su razón de ser?

La diferencia fundamental está en que la esfera es una entidad ideal, una pura esencia, que permite ser considerada en sí misma, sin conexión con otras cosas posibles: "También la noción de la esfera en general es incompleta o abstracta, es decir, no se considera de ella más que la esencia de la esfera en general o en teoría, sin considerar las circunstancias singulares y, por consiguiente, ella no contiene de ningún modo lo que es requisito para la existencia de una determinada esfera" ${ }^{16}$. Por el contrario, no hay una noción genérica del yo, porque "el susodicho yo, o lo que le corresponde en cada sustancia individual" ${ }^{17}$ es un ser completo. La completud es el requisito fundamental del yo y de la sustancia individual concebida a la manera del yo.

La calificación de sustancia individual como completa, en el marco del Discurso de metafisica y la subsiguiente correspondencia con Arnauld, marca un

\footnotetext{
${ }^{13}$ Carta de Arnauld a Leibniz, 13. 05. 1686, OFC 14, 29-30.

${ }^{14}$ Arnauld, 1986, 59.

${ }^{15}$ Observaciones de Leibniz a una carta de Antoine Arnauld, 14. 07. 1686, OFC 14, 36-37.

${ }^{16}$ Ibid., 37.

${ }^{17}$ Carta de Leibniz a Arnauld, 28.11.1686/8.12.1686, OFC 14, 80.
} 
viraje en la metafísica leibniziana. Efectivamente, en la Disputatio de principio individui (1663), la sustancia es calificada como tota, lo que significa que forma una unidad indivisible con sus accidentes y que se constituye a sí misma, a se, a partir de su propia entidad. Al introducir el término 'completo' como calificativo de la sustancia, Leibniz mantiene la idea de que el ente real se constituye por un dinamismo intrínseco del posible, que en la idea de un individuo considerado en el estado de posibilidad se encuentra la "razón y fundamento" de todos sus estados. Si embargo, el calificativo de completo añade un nuevo aspecto, que es la relación de composibilidad con todos los demás seres existentes. La completud, interpretada en términos leibnizianos, lleva al límite la relación de composibilidad y coexistencia de los seres: el yo es completo en la medida en que es el todo bajo un determinado punto de vista, conteniendo tanto orden como el mundo del que es una "parte total". El artículo 14 del Discurso de metafísica es muy preciso en este sentido: una sustancia individual es una especie de emanación que acompaña al pensamiento divino, en cuanto que este último tiene una percepción particular del "sistema general de los fenómenos" que constituye la materia fenoménica del propio mundo ${ }^{18}$. Un individuo es, por tanto, "el perfil del universal" o la realización de una nueva posibilidad del mundo.

Desarrollando y profundizando una línea ya apuntada en carta a Foucher de 1675 , donde se afirma que hay dos verdades igualmente primitivas y mutuamente independientes, es decir, que yo pienso y que muchas cosas son pensadas por mí, Leibniz establece una conexión muy estrecha entre el yo y su mundo. Lejos de ser una entidad atómica y solipsista, el individuo es miembro de un mundo común, que le corresponde expresar. En cuanto al mundo, constituye un marco de relaciones que forman una serie ordenada de fenómenos, que se desplieganen en una infinidad de series, las cuales se desarrollan siguiendo un mismo principio de orden. De ahí que haya una correspondencia perfecta o "relación regulada" entre los fenómenos de cada una de las cosas que componen el mundo actual. A este régimen intensificado de orden lo llama Leibniz 'expresión', que "es común a todas las formas" y se define como "una relación constante y reglada entre lo

\footnotetext{
18 "Dios, en efecto, hace girar, por así decir, por todos los lados y de todas las maneras el sistema general de los fenómenos que considera oportuno producir para manifestar su gloria y mira todos los aspectos del mundo de todas las maneras posibles, puesto que no existe relación alguna que escape a su omnisciencia; el resultado de cada visión del universo, en el que éste comparece como contemplado desde un determinado lugar, es una sustancia que expresa el universo conforme a esa visión, con tal que Dios considere conveniente hacer efectivo su pensamiento y producir esa sustancia." (OFC 2, 175-176).
} 
que se puede decir de una y de la otra"19 de las cosas conectadas por la expresión. La expresión es el gran operador de la armonía universal, que es la condición radical de todo ser y acción.

El yo es una expresión singular del mundo actual. Por consiguiente, su identidad no consiste en un fondo sustancial inmutable, sino en una serie ordenada de cambios según un principio interno de orden. Es éste un punto focal de divergencia entre Leibniz y Arnauld. En efecto, para Arnauld mi yo es una entidad permanente cuya naturaleza sustancial es indiferente a los múltiples accientes que puedan afectarla. El hecho, por ejemplo, de hacer o no hacer un viaje en nada afecta a la noción individual de mi yo: "Estoy seguro de que en tanto que pienso, yo soy yo; pues no puedo pensar que yo no existo, ni que yo no soy yo. Pero puedo pensar que haré tal viaje o que no lo haré, estando segurísimo de que ni lo uno ni lo otro impedirá que yo sea yo. Estoy convencido de que ni lo uno ni lo otro está contenido en la noción individual de 'yo'”20. Leibniz adopta una posición drásticamente crítica respecto a la tesis de Arnauld, al considerar que se trata de una idea preconcebida: "Es un prejuicio ["prévention»] que no hay que confundir con una noción o conocimiento distinto." 21

El procedimiento leibniziano es, por tanto, diametralmente opuesto al de Arnauld: en vez de situar el yo como base de la noción vulgar de sustancia, es ésta la que debe adaptarse al modo de efectividad del yo, a su realidad. El yo es una forma princeps de auténtica unidad; es éste el tema central de la carta a Arnauld del 30 de abril de 1687, donde se afirma el carácter derivado de la extensión, no reconociéndole el estatuto de sustancia, en cuanto que expresa sólo el estado presente, y no el futuro ni el pasado, y sobre todo por carecer de unidad debido a la uniformidad e indistinción de sus partes. Mientras que una verdadera unidad, como lo es toda cosa animada, "contiene un mundo de diversidades en una verdadera unidad"22. Una verdadera unidad se despliega en el tiempo según un orden invisible y algo laberíntico, en donde un aspecto casi imperceptible puede desviar el curso de una vida. Por consiguiente, la narrativa del yo asume la forma

${ }^{19}$ Carta de Leibniz a Arnauld, 9. 10. 1687, OFC 14, 120.

${ }^{20}$ Carta de Arnauld a Leibniz, OFC 14, 30.

${ }^{21}$ Observaciones de Leibniz a una carta de Antoine Arnauld, 14. 07. 1686, OFC 14, 43.

${ }^{22}$ OFC 14, 106. 
de una biografía compuesta de una unidad con una infinidad de acontecimientos diversos y una red infinita de relaciones.

\section{La subjetividad monadológica}

La monadología, en el sentido de un marco general de inteligibilidad, es la culminación de la práctica filosófica de Leibniz, en particular en lo referente a la subjetividad.

La mónada es una unidad real, distinta de las unidades arbitrarias de la matemática. Leibniz no abandona enteramente el vocabulario de la sustancia completa, pero procede a calificar la sustancia preferentemente como simple: "La mónada, de la que vamos a hablar aquí, no es sino una sustancia simple que entra en los compuestos" (Monadología, art. 1, OFC 2, 328).

A diferencia de una parte de la exégesis leibniziana, que tiende a hipostasiar a las mónadas, por considerar que constituyen un mundo aparte, entendemos que Leibniz establece una correlación muy fuerte entre los simple y lo compuesto. De hecho, las mónadas entran en los compuestos y, más aún, son "los elementos de las cosas" (Monadología, art. 3, OFC 2, 328), interpretando el elemento en el sentido preciso de principio primero de constitución de una cosa: "aquello de lo que algo está primariamente compuesto" ${ }^{23}$.

En su calidad de sustancia, la mónada es "un ser capaz de acción” (OFC 2, 344), que consiste únicamente en las "percepciones y sus cambios" (Monadología, art. 17, OFC 2, 330). A diferencia de Descartes ${ }^{24}$ e incluso de Locke ${ }^{25}$, Leibniz asume la percepción como sinónimo de actividad vital, mediante la cual el ser vivo conecta con el mundo y ejerce su espontaneidad. Ahora bien, como toda verdadera acción, esa actividad es inmanente y resulta de un "principio interno".

\footnotetext{
${ }^{23}$ Aristóteles, Metafísica 1014 a.

24 “Todos los modos de pensar que observamos en nosotros pueden ser referidos a dos formas generales: una consiste en percibir mediante el entendimiento y la otra en determinarse mediante la voluntad" (Descartes, Principes, parte I, art. 32, AT IX, 39; trad. G. Quintás, Madrid, Alianza, 1995, pp. 40-41).

25 "La percepción es la primera facultad de la mente que se ocupa de nuestras ideas" (Locke, 1999, p. 126; Ensayo sobre el entendimiento humano, trad. S. Rábade y E. García, Madrid, Editora Nacional, 1980, p. 219).
} 
En la definición leibniziana, la percepción es un "estado transitorio", lo que indica que es el eslabón de un continuum, que se inscribe en un proceso continuo de cambios. Se trata de un proceso gradual e insensible, luego el marco general de los fenómenos que se nos presentan no cambia abruptamente: lo que se produce son pequeñas variaciones en el modo en que somos afectados o nos relacionamos con las cosas. Leibniz lo expresa con gran precisión en el artículo 12 de la Monadología: "Pero también es preciso que, además del principio del cambio, haya un detalle de lo que cambia, que efectúe, por así decir, la especificación y la variedad de las sustancias simples." (OFC 2, 329).

Es sin duda muy relevante que aquello que constituye la singularidad distintiva de las mónadas sea una variación mínima (punto de variación) del marco fenoménico: una atención especial a una sonrisa, al amarillear de una hoja, a la modulación de un sonido. La singularidad se opera, entonces, por subjetivación, por un modo peculiar de afección y de relación con el mundo, que se expresa por la disposición hacia una determinada serie de percepciones en lugar de otra. Esta disposición, que Leibniz designará como affectus, en la terminología monadológica se denomina apetición y se define como "la acción del principio interno que realiza el cambio o paso de una percepción a otra" (Monadología, art. 15, OFC 2, 329).

La razón de ser de la apetición se debe a la diferencia entre lo buscado y lo percibido. El acto perceptivo aprehende un número limitado de objetos, pero apunta al todo, al infinito, tal como está expresado en el artículo 60 de la Monadología: "No es por el objeto, sino por la modificación del conocimiento del objeto por lo que las mónadas son limitadas. Todas se dirigen confusamnte al infinito, al todo, pero son limitadas y se distinguen por los grados de las percepciones distintas" (OFC 2, 336). Por consiguiente, la apetición es el propio dinamismo interno de la vida perceptiva, no un determinado tipo de instancia exterior. Su función es inscribir la tendencia en el estado actual de la mónada, convirtiéndolo en pasajero, en tensión hacia nuevas percepciones. Así es, el apetito de la mónada "siempre obtiene algo" (Monadología, art. 15, OFC 2, 329), pero no alcanza nunca al infinito hacia el cual tiende, debido al margen de confusión presente en cada percepción. Por tanto, confusión e infinitud de la percepción son indisociables. La confusión viene de la unión de la mónada con un cuerpo propio, a través del cual participa en el orden universal.

Efectivamente, la percepción es un fenómeno psíquico que sucede espontáneamente en la propia alma y no en el cerebro o en las partes sutiles del cuerpo, 
lo cual no significa que el alma sea una entidad solipsista, al ser naturalmente representativa de los fenómenos exteriores gracias a su vinculación con un cuerpo. Tal como se dice en el Nuevo sistema de la naturaleza, el alma tiene una "naturaleza representativa (capaz de expresar a los seres externos con relación a sus órganos)" (OFC 2, 247). Esta sorprendente atribución de órganos al alma adquiere sentido en el contexto de un parágrafo donde se afirma que "el punto de vista del alma”, según el cual ella expresa el universo, está en la "masa organizada", esto es, en el cuerpo orgánico (Ibid.). Manteniéndose fiel a su tesis de que el cuerpo y el alma se influyen directamente entre sí, Leibniz lleva al límite su acomodación y expresión mutuas. De ahí la afirmación de que existe una "unión física del alma y el cuerpo" (OFC 2, 344), esto es, que se ajustan naturalmente entre sí en su funcionamiento.

El cuerpo tiene un papel mediador entre el alma y el mundo externo, cuya representación es función del alma: "y como este cuerpo expresa todo el universo, por la conexión de toda la materia en lo lleno, el alma representa también todo el universo, al representar este cuerpo que le pertenece de un modo particular" (Monadología, art. 62, OFC 2, 337). ¿De qué manera sucede esto? El cuerpo es afectado por los objetos más próximos y, a través de ellos, por todos los cuerpos del universo, de los cuales recibe impresiones. En realidad, el cuerpo orgánico es una máquina natural o mecanismo perfecto e infinito, pero, además, es también una estructura sensible de afección. Según el léxico leibniziano, el cuerpo es afectado por las acciones de los otros cuerpos y recibe de ellos impresiones, que podemos entender como afecciones físicas. En el artículo 61 de la Monadología, Leibniz dice que "de esa manera cada cuerpo es afectado no sólo por aquellos con los que está en contacto, y de algún modo siente todo lo que les ocurre, sino que también, a través de ellos, siente a los que tocan a los primeros con los que está inmediatamente en contacto; de todo esto se sigue que esta comunicación llega a cualquier distancia" (OFC 2, 336-337). Ahora bien, ser afectado y sentir pertenecen al ámbito de la sensibilidad física. Pero tal vez sea en el artículo 4 de los Principios de la naturaleza y de la gracia donde el filósofo de la armonía aclara mejor la continuidad entre lo orgánico y lo psíquico en la vida perceptiva: "Pero cuando la mónada tiene órganos tan ajustados que mediante ellos hay relieve y distinción en las impresiones que reciben y, por consiguiente, en las percepciones que las representan (...)" (OFC 2, 345). Las mónadas se acomodan a las impresiones orgánicas y las expresan bajo el modo de la percepción, otorgándoles una significación psíquica, que amplía su significado físico. 
Leibniz no niega la posibilidad de existencia de espíritus puros, totalmente separados de la materia. Sin embargo, tales espíritus serían entidades solipsistas, incapaces de comunicar y de participar en cualquier tipo de orden, una especie de "desertores del orden general" ${ }^{26}$. Por consiguiente, el mejor de los mundos "será siempre un sistema de cuerpos (es decir, de cosas ordenadas según los lugares y los tiempos) y de almas que representan y perciben los cuerpos, y conforme a los cuales los cuerpos son gobernados en buena medida." (Ensayos de Teodicea, art. 200, OFC 10,238). La necesidad del cuerpo está en que es un requisito de orden para el alma: "en efecto, toda sustancia simple tiene un cuerpo orgánico que le corresponde, de otro modo no tendría ninguna relación establecida con las otras en el universo y no podría actuar ni padecer ordenadamente." 27

\section{Conclusión}

La inscripción del yo en el núcleo de la sustancia contribuye poderosamente a la renovación de la metafísica leibniziana, superando el carácter abstracto y nominal de la noción aristotélico-escolástica de la sustancia. El yo se revela como la unidad de un proceso que se desarrolla bajo forma de una secuencia ordenada de estados y, simultáneamente, como un punto de variación del mundo actual.

La filosofía monadológica, que culmina el pensamiento de Leibniz, proporciona el marco propio de una comunidad de seres vivos, cuya actividad consiste en la percepción, que es a la vez un tipo de acción inmanente y una forma de comunicación con el entorno.

La vida se manifiesta como un flujo espontáneo de percepciones, muchas de las cuales son pequeñas percepciones, que tienen la función básica de garantizar la continuidad de un mismo ser vivo. Leibniz califica de insensibles a estas pequeñas percepciones de las cuales no nos apercibimos porque se sitúan a un nivel

\footnotetext{
26 "Solamente Dios está por encima de toda la materia, puesto que es el autor de ella, pero las criaturas exentas o emancipadas de la materia estarían al mismo tiempo desligadas de la ligazón universal, y serían como desertoras del orden general. " (Consideraciones sobre los principios de vida, OFC 8, 516-517).

${ }_{27}$ Consecuencias metafísicas del principio de razón, C, 14.
} 
subconsciente del psiquismo. Así, la conciencia no pasa de ser una fulguración puntual, discontinua, de la vida perceptiva.

El yo es la expresión más elevada de la vida, pero no es lo dado primordial. El estrato fundador de la vida es el de un sí mismo (soi), previo a la conciencia, a partir del cual, por un acto reflexivo de apercepción, se constituye el yo. Muy significativamente, Leibniz invierte la relación lockiana entre el sí mismo y la conciencia: mientras que para Locke el sí mismo era el resultado de la actividad consciente, en Leibniz es la conciencia la que emerge como fulguración de un sí mismo primordial.

La subjetividad comporta grados, según una dinámica expresiva, en donde lo más elevado encaja en las formas elementales, y cuyo significado no debe subestimarse, como insiste Leibniz a propósito de la distinción entre percepción y apercepción, para señalar el gran error de los cartesianos: "Y precisamente en este punto los cartesianos han caído en un grave error, por no haber tenido en cuenta las percepciones de las que no nos apercibimos." 28

${ }^{28}$ Monadología, art. 14, OFC 2, 329. 


\section{Referencias bibliográficas}

Arnauld, A., (1986), Des vraies et des fausses idées. Paris: Fayard.

Descartes, R., Oeuvres publiées par Charles Adam et Paul Tannery. Paris: Vrin.

GiL, F., (1996), Tratado da evidência. Lisboa: Imprensa Nacional-Casa da Moeda.

La Forge, L. (1664) Traité de l'esprit de l'homme et de ses facultés ou fonctions et de son union avec le corps, Amsterdam.

Locke, J., (1999), An essay concerning human understanding. Pennsilvania: Pennsilvania State University.

Malebranche, N., (1974), Réflexions sur la prémotion physique. Cuvres Complètes de Malebranche, ed. Robinet, tome. Paris: Vrin.

Malebranche, N., (1991), Entretiens sur la métaphysique et sur la religion. Euvres Complètes de Malebranche, ed. Robinet, tomes XII-XIII. Paris: Vrin.

Recibido: 27/10/2016

Aceptado: 16/11/2016

Este trabajo se encuentra bajo una licencia de Creative Commons Reconocimiento-NoComercial-SinObraDerivada 4.0 Internacional

\section{c) (1) $\Theta$


